\title{
Protecting entanglement from decoherence using weak measurement and quantum measurement reversal
}

\author{
Yong-Su Kim ${ }^{\dagger}$, Jong-Chan Lee ${ }^{\dagger}$, Osung Kwon and Yoon-Ho Kim
}

\begin{abstract}
Decoherence, often caused by unavoidable coupling with the environment, leads to degradation of quantum coherence'. For a multipartite quantum system, decoherence leads to degradation of entanglement and, in certain cases, entanglement sudden death ${ }^{2,3}$. Tackling decoherence, thus, is a critical issue faced in quantum information, as entanglement is a vital resource for many quantum information applications including quantum computing ${ }^{4}$, quantum cryptography ${ }^{5}$, quantum teleportation $^{6-8}$ and quantum metrology ${ }^{9}$. Here, we propose and demonstrate a scheme to protect entanglement from decoherence. Our entanglement protection scheme makes use of the quantum measurement itself for actively battling against decoherence and it can effectively circumvent even entanglement sudden death.
\end{abstract}

One way to cope with decoherence is to make use of entanglement distillation protocols by which a pure maximally entangled state may be obtained from multiple copies of partially decohered states ${ }^{4,10-14}$. Note, however, that it is impossible to obtain an entangled state from copies of fully decohered (that is, separable) states by applying entanglement distillation ${ }^{15}$. Another method to deal with decoherence is to rely on the so-called decoherence-free subspace $^{16,17}$. The decoherence-free subspace, however, requires the interaction Hamiltonian to have an appropriate symmetry, which might not always be present. The quantum Zeno effect may also be used to suppress decoherence ${ }^{18,19}$ as well as to generate entanglement ${ }^{20}$ under some specific situations.

Our scheme for protecting entanglement from decoherence is based on the fact that weak quantum measurement can be reversed. The reversibility of weak quantum measurement was originally discussed in the context of quantum error correction ${ }^{21}$ and was demonstrated for a single superconducting qubit and a single photonic qubit ${ }^{22-24}$. Recently, it was shown that weak measurement and quantum measurement reversal can effectively suppress amplitude-damping decoherence for a single qubit $^{25,26}$. Here, we experimentally demonstrate a scheme for protecting entanglement from amplitude-damping decoherence using weak measurement and quantum measurement reversal. The scheme can reduce or even completely nullify the effect of decoherence as evidenced by increased concurrence of the two-qubit system.

Consider a two-level quantum system $(\mathrm{S})$ whose computational bases are $|0\rangle_{S}$ and $|1\rangle_{S}$. The environment (E) is initially at $|0\rangle_{\mathrm{E}}$. Amplitude-damping decoherence, in which a particular computational basis state is irreversibly and probabilistically transferred to the other, results from state-dependent coupling
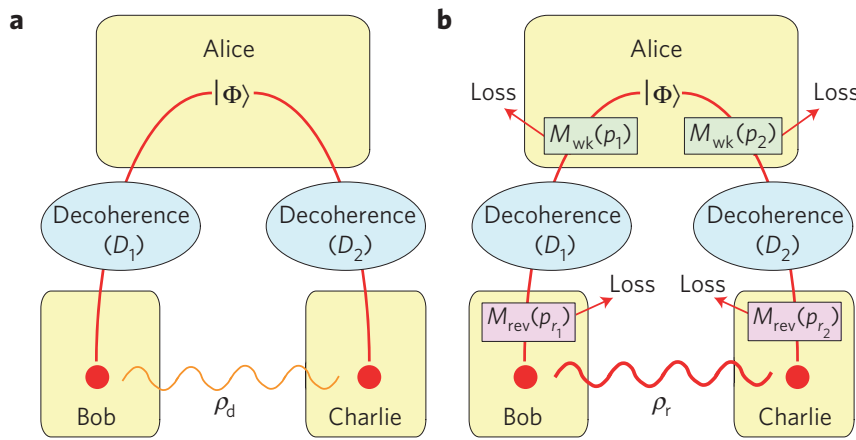

Figure 1 | Scheme for protecting entanglement from decoherence using weak measurement and quantum measurement reversal. a, Owing to decoherence $D_{1}$ and $D_{2}$ in the quantum channels, Bob and Charlie end up sharing the quantum state $\rho_{\mathrm{d}}$, which is either less entangled than $|\Phi\rangle$ or not entangled at all owing to ESD. b. Protecting entanglement from decoherence using weak measurement and quantum measurement reversal. Alice first carries out weak measurement $\left(M_{\mathrm{wk}}\right)$ on $|\Phi\rangle$ before distribution. Bob and Charlie, on receiving the qubits, carry out reversing measurement $\left(M_{\text {rev }}\right)$. The resulting shared quantum state $\rho_{\mathrm{r}}$ can be made as close to the original $|\Phi\rangle$ by choosing $\left\{p_{1}, p_{2}\right\}$ and $\left\{p_{\mathrm{r}_{1}}, p_{\mathrm{r}_{2}}\right\}$ properly.

of the system qubit to the environment and is described by the following quantum map,

$$
\begin{aligned}
|0\rangle_{\mathrm{S}}|0\rangle_{\mathrm{E}} & \rightarrow|0\rangle_{\mathrm{S}}|0\rangle_{\mathrm{E}} \\
|1\rangle_{\mathrm{S}}|0\rangle_{\mathrm{E}} & \rightarrow \sqrt{\bar{D}}|1\rangle_{\mathrm{S}}|0\rangle_{\mathrm{E}}+\sqrt{D}|0\rangle_{\mathrm{S}}|1\rangle_{\mathrm{E}}
\end{aligned}
$$

where $0 \leq D \leq 1$ is the magnitude of the decoherence and $\bar{D}=1-\bar{D}$ (ref. 4). Amplitude-damping decoherence is highly relevant for many practical qubit systems. For instance, amplitudedamping decoherence is caused by photon loss for the vacuumsingle-photon qubit, by spontaneous decay for the atomic energy level qubit and by zero-temperature energy relaxation for the superconducting qubit.

We now investigate how the decoherence map of equation (1) affects a two-qubit entangled state. In particular, we consider a quantum communication scenario depicted in Fig. 1a where Alice prepares a two-qubit entangled state $|\Phi\rangle=\alpha|00\rangle_{\mathrm{S}}+\beta|11\rangle_{\mathrm{S}}$ $\left(\alpha \alpha^{*}+\beta \beta^{*}=1\right)$ and distributes the qubits to Bob and Charlie through quantum channels with decoherence $D_{1}$ and $D_{2}$. The discussion, however, applies equally well to other types of two-qubit entangled state and physical qubit of stationary nature. 

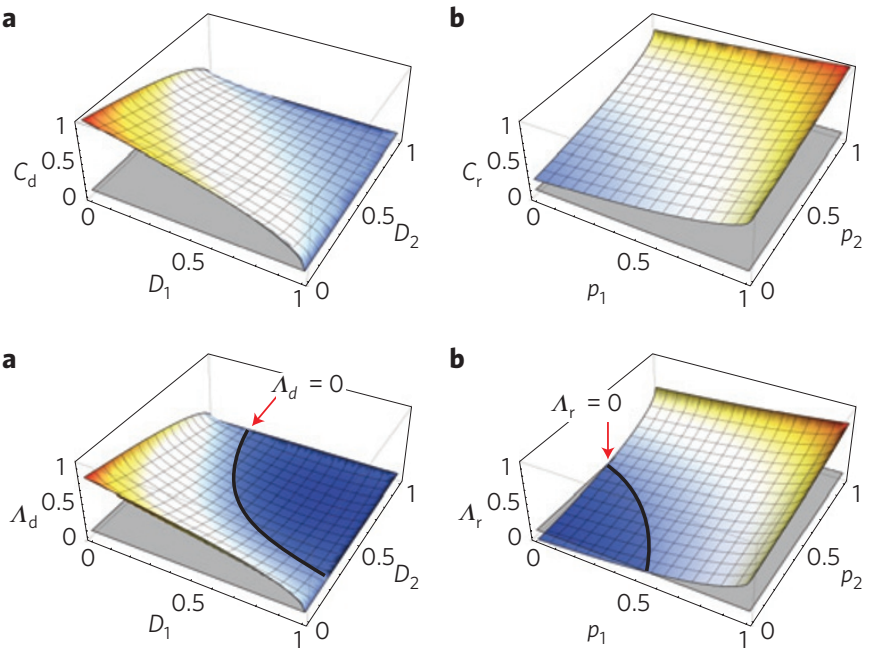

Figure 2 | Theoretical estimation of concurrence change as functions of decoherence and weak measurement. The two quantum channels have different decoherence $D_{1}$ and $D_{2}$. a-d, Plots for the maximally entangled state $|\Phi\rangle(|\alpha|=|\beta|)(\mathbf{a}, \mathbf{b})$ and for the non-maximally entangled state $|\Phi\rangle$ $(|\alpha|<|\beta|$ with $|\alpha|=0.42)$ (c,d). Entanglement degradation due to decoherence is shown in $\mathbf{a}$ and $\mathbf{c}$. Weak measurement with strength $p_{1}$ and $p_{2}$ and the optimal reversing measurement can circumvent decoherence as shown in $\mathbf{b}$ and $\mathbf{d}$. Plots $\mathbf{b}$ and $\mathbf{d}$ are for $D_{1}=0.6$ and $D_{2}=0.8$. The horizontal planes represent zero concurrence. Note that c shows ESD and d shows that even ESD-causing decoherence can be circumvented with weak measurement of sufficient strength and corresponding optimal reversing measurement.

Although the initial state was a pure two-qubit entangled state $|\Phi\rangle$, owing to decoherence, Bob and Charlie now share the twoqubit quantum state $\rho_{\mathrm{d}}$ given as

$$
\rho_{\mathrm{d}}=\left(\begin{array}{cccc}
|\alpha|^{2}+|\beta|^{2} D_{1} D_{2} & 0 & 0 & \sqrt{\bar{D}_{1} \bar{D}_{2}} \alpha^{*} \beta \\
0 & D_{1} \bar{D}_{2}|\beta|^{2} & 0 & 0 \\
0 & 0 & D_{2} \bar{D}_{1}|\beta|^{2} & 0 \\
\sqrt{\bar{D}_{1} \bar{D}_{2}} \alpha \beta^{*} & 0 & 0 & \bar{D}_{1} \bar{D}_{2}|\beta|^{2}
\end{array}\right)
$$

where $\bar{D}_{\mathrm{k}}=1-D_{\mathrm{k}}(\mathrm{k}=1,2)$. The effect of decoherence $D_{1}$ and $D_{2}$ on the initial state $|\Phi\rangle$ can then be investigated by evaluating concurrence $C_{\mathrm{d}}$ (which quantifies the amount of entanglement) of the shared quantum state $\rho_{\mathrm{d}}$ (ref. 27), which is calculated to be

$$
C_{\mathrm{d}}=\max \left\{0, \Lambda_{\mathrm{d}} \equiv 2 \sqrt{\bar{D}_{1} \bar{D}_{2}}|\beta|\left(|\alpha|-\sqrt{D_{1} D_{2}}|\beta|\right)\right\}
$$

Note that $C_{\mathrm{d}}=\Lambda_{\mathrm{d}}$ if $\Lambda_{\mathrm{d}}>0$. It is clear from equation (2) that the stronger the decoherence, $D_{\mathrm{k}} \rightarrow 1(\mathrm{k}=1,2)$, the smaller the concurrence $C_{\mathrm{d}}$. When the decoherence is strongest $D_{\mathrm{k}}=1(\mathrm{k}=$ $1,2)$, concurrence $C_{\mathrm{d}}$ becomes zero, meaning that the two-qubit system has become fully separable. Furthermore, it is interesting to point out that, for a particular initial two-qubit state $|\Phi\rangle=\alpha|00\rangle_{\mathrm{S}}+$ $\beta|11\rangle_{\mathrm{S}}$ with $|\beta| \geq|\alpha|$, the decoherence causes entanglement sudden death (ESD) in which $C_{\mathrm{d}}=0$ although decoherence is not at its maximum: $C_{\mathrm{d}}=0$ if decoherence $\sqrt{D_{1} D_{2}} \geq|\alpha / \beta|$ (ref. 3).

Let us now describe our scheme, depicted in Fig. 1b, to protect entanglement from decoherence by making use of weak measurement and quantum measurement reversal. Before the system qubits undergo decoherence (that is, coupling to the environment), they are subject to weak measurement $\left(M_{\mathrm{wk}}\right)$, which partially collapses the state towards $|0\rangle_{\mathrm{S}}$ (refs 22-24). The

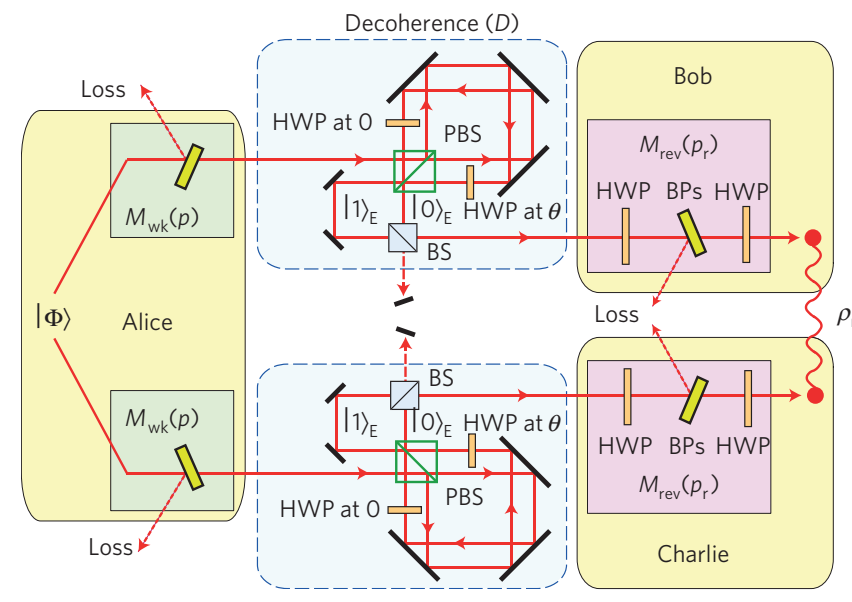

Figure 3 | Schematic of the experiment. The initial two-qubit state $|\Phi\rangle$ is prepared with the two-photon polarization state. Weak measurement $\left(M_{\mathrm{wk}}\right)$ and the optimal reversing measurement $\left(M_{\mathrm{rev}}\right)$ are carried out on the polarization qubit using Brewster-angle glass plates (BPs) and half-wave plates ${ }^{24}$ (HWPs). Amplitude-damping decoherence is introduced to the polarization qubit using an interferometer ${ }^{26}$. See Methods for details.

two-qubit weak measurement can be written as a non-unitary quantum operation

$$
M_{\mathrm{wk}}\left(p_{1}, p_{2}\right)=\left(\begin{array}{cc}
1 & 0 \\
0 & \sqrt{1-p_{1}}
\end{array}\right) \otimes\left(\begin{array}{cc}
1 & 0 \\
0 & \sqrt{1-p_{2}}
\end{array}\right)
$$

where $p_{1}$ and $p_{2}$ are the weak measurement strengths ${ }^{24}$. As the computational basis state $|0\rangle_{\mathrm{S}}$ does not couple to the environment as shown in equation(1), the system qubits after the weak measurement are less vulnerable to decoherence.

After the decoherence quantum channel, Bob and Charlie carry out quantum measurement reversal operations on the qubits. The two-qubit reversing measurement $\left(M_{\text {rev }}\right)$ is a non-unitary operation that can be written as

$$
M_{\text {rev }}\left(p_{\mathrm{r}_{1}}, p_{\mathrm{r}_{2}}\right)=\left(\begin{array}{cc}
\sqrt{1-p_{\mathrm{r}_{1}}} & 0 \\
0 & 1
\end{array}\right) \otimes\left(\begin{array}{cc}
\sqrt{1-p_{\mathrm{r}_{2}}} & 0 \\
0 & 1
\end{array}\right)
$$

where $p_{\mathrm{r}_{1}}$ and $p_{\mathrm{r}_{2}}$ are the strengths of the reversing measurement. The optimal reversing measurement strength that gives the maximum amount of entanglement of the two-qubit state $\rho_{\mathrm{r}}$ is calculated to be $p_{\mathrm{r}_{\mathrm{k}}}=p_{\mathrm{k}}+D_{\mathrm{k}} \bar{p}_{\mathrm{k}}$, where $\bar{p}_{\mathrm{k}}=1-p_{\mathrm{k}}(\mathrm{k}=1,2 ;$ refs 25,26$)$. Assuming that the reversing measurement is optimal, the two-qubit state now shared by Bob and Charlie (after the sequence of weak measurement, decoherence and reversing measurement) is given as

$$
\rho_{\mathrm{r}}=\frac{1}{\mathcal{A}}\left(\begin{array}{cccc}
|\alpha|^{2}+|\beta|^{2} D_{1} D_{2} \bar{p}_{1} \bar{p}_{2} & 0 & 0 & \alpha^{*} \beta \\
0 & D_{1} \bar{p}_{1}|\beta|^{2} & 0 & 0 \\
0 & 0 & D_{2} \bar{p}_{2}|\beta|^{2} & 0 \\
\alpha \beta^{*} & 0 & 0 & |\beta|^{2}
\end{array}\right)
$$

where $\mathcal{A}=1+\left\{D_{1} \bar{p}_{1}\left(1+D_{2} \bar{p}_{2}\right)+D_{2} \bar{p}_{2}\right\}|\beta|^{2}$. The concurrence $C_{\mathrm{r}}$ of the two-qubit state $\rho_{\mathrm{r}}$ shared by Bob and Charlie is then calculated to be

$$
C_{\mathrm{r}}=\max \left\{0, \Lambda_{\mathrm{r}} \equiv \frac{2|\beta|\left(|\alpha|-\sqrt{D_{1} D_{2} \bar{p}_{1} \bar{p}_{2}}|\beta|\right)}{1+\left\{D_{1} \bar{p}_{1}\left(1+D_{2} \bar{p}_{2}\right)+D_{2} \bar{p}_{2}\right\}|\beta|^{2}}\right\}
$$

where $C_{\mathrm{r}}=\Lambda_{\mathrm{r}}$ if $\Lambda_{\mathrm{r}}>0$.

We can draw two important conclusions from the result in equation (3). First, $C_{\mathrm{r}}$ is always larger than $C_{\mathrm{d}}$, which means that 


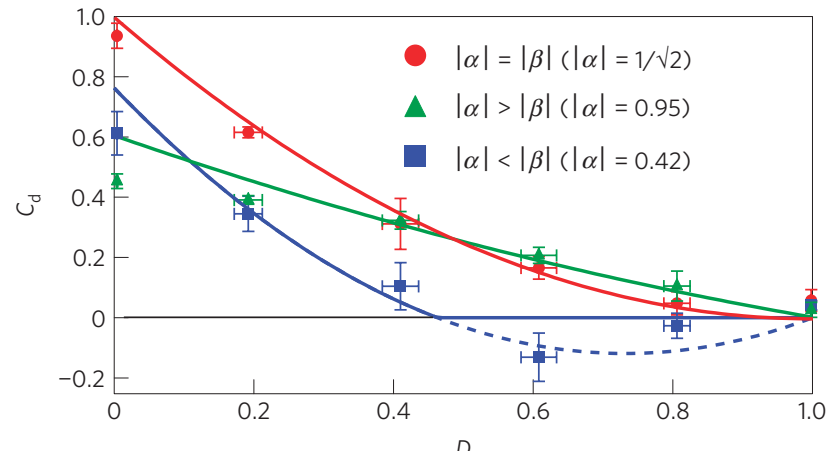

b

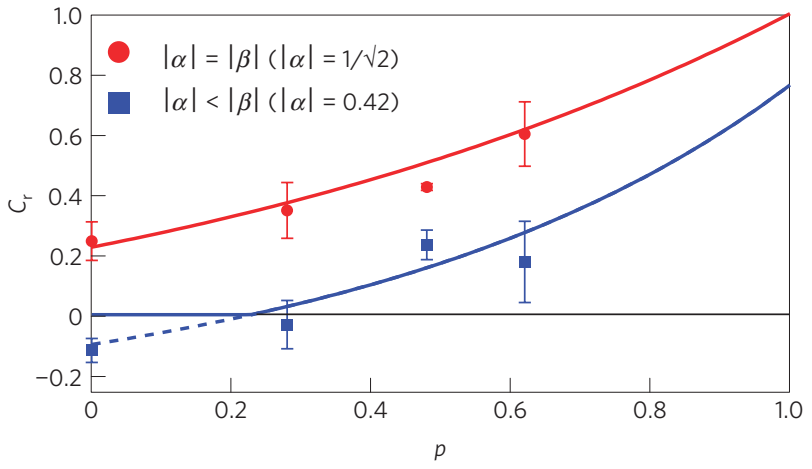

Figure 4 | Experimental data for protecting entanglement from decoherence using weak measurement and quantum measurement reversal. a, As $D$ is increased, the state $\rho_{\mathrm{d}}$ loses entanglement gradually. b, Carrying out $M_{\mathrm{wk}}(p)$ and the corresponding optimal $M_{\mathrm{rev}}\left(p_{\mathrm{r}}\right)$ enables distribution of entanglement under strong decoherence $(D=0.6)$. Negative values are $\Lambda_{d}$ for $\mathbf{a}$ and $\Lambda_{\mathrm{r}}$ for $\mathbf{b}$. The error bars represent the statistical error of \pm 1 standard deviation.

weak measurement and quantum measurement reversal indeed can be used for protecting entanglement from decoherence. It is possible to achieve $\rho_{\mathrm{r}} \rightarrow|\Phi\rangle$ provided that the strength of the weak measurement $p_{\mathrm{k}}$ and that of the corresponding optimal reversing measurement $p_{\mathrm{r}_{\mathrm{k}}}(\mathrm{k}=1,2)$ are sufficiently strong. Second, even for the ESD condition (that is, for the initial state $|\Phi\rangle$ with $|\beta|>|\alpha|, C_{\mathrm{d}}=0$ if decoherence $\left.\sqrt{D_{1} D_{2}} \geq|\alpha / \beta|\right)$, by applying the entanglement protection protocol with proper weak measurement $\left(\bar{p}_{1} \bar{p}_{2} \leq\left(1 / D_{1} D_{2}\right)|\alpha / \beta|^{2}\right)$ and the corresponding optimal reversing measurement, Bob and Charlie are able to share some entanglement.

In Fig. 2, we show how $C_{\mathrm{d}}\left(\Lambda_{\mathrm{d}}\right)$ and $C_{\mathrm{r}}\left(\Lambda_{\mathrm{r}}\right)$ behave for two particular initial states under different decoherence, weak measurement and corresponding optimal reversing measurement. It is clear that decoherence affecting the two qubits independently and at different magnitudes can be circumvented by exploiting weak measurement and quantum measurement reversal.

Let us now describe the experimental demonstration of protecting entanglement from decoherence using weak measurement and quantum measurement reversal for an entangled two-qubit system. The experimental set-up consisting of three principle sections (weak measurement, decoherence and reversing measurement) is schematically shown in Fig. 3. Details of the experimental set-up are described in the Methods section. For a clear demonstration, we consider identical decoherence and weak measurement for both qubits, that is, $D_{1}=D_{2}=D$ and $p_{1}=p_{2}=p$. In our experiment, the system qubits are realized with the single-photon polarization state; $|0\rangle_{S}$ and $|1\rangle_{S}$ refer to horizontal and vertical polarization, respectively.

We first examine the effect of decoherence $D$ on the initial twoqubit entangled state $|\Phi\rangle=\alpha|00\rangle_{\mathrm{S}}+\beta|11\rangle_{\mathrm{S}}$ without introducing

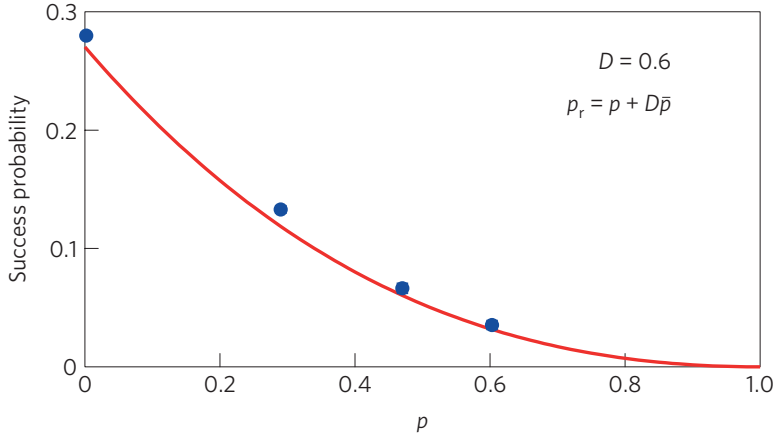

Figure 5 | The success probability as a function of weak measurement strength. Filled circles represent experimental data and the solid line is calculated according to equation (4).

the weak measurement and the reversing measurement. The resulting two-qubit state $\rho_{\mathrm{d}}$ is reconstructed with quantum state tomography ${ }^{24,26}$ and concurrence $C_{\mathrm{d}}$ is then evaluated. Figure $4 \mathrm{a}$ shows the experimental data for three input state conditions $(|\alpha|=|\beta|,|\alpha|>|\beta|$ and $|\alpha|<|\beta|)$ as a function of decoherence $D$. In all cases, the data show loss of entanglement due to decoherence. Note that, for $|\alpha|<|\beta|$, ESD occurs as expected in equation (2).

We now test whether our entanglement protection scheme, based on weak measurement and quantum measurement reversal, can indeed circumvent decoherence. To demonstrate the scheme's ability to protect entanglement even under severe decoherence, we chose $D=0.6$, at which ESD is demonstrated for the initial state with $|\alpha|<|\beta|$ as shown in Fig. 4a. Figure $4 \mathrm{~b}$ shows concurrence $C_{\mathrm{r}}$ of the two-qubit state $\rho_{\mathrm{r}}$ as a function of weak measurement strength $p$ for two sets of initial states: $|\alpha|=|\beta|$ and $|\alpha|<|\beta|$. For each weak measurement strength $p$, the optimal reversing measurement strength $p_{\mathrm{r}}=p+D \bar{p}$ was chosen. For the maximally entangled two-qubit state $(|\alpha|=|\beta|)$, the weak measurement and the reversing measurement indeed suppress two-qubit decoherence so that $C_{\mathrm{r}}>C_{\mathrm{d}}$ and $C_{\mathrm{r}} \rightarrow 1$ as $p \rightarrow 1$. Furthermore, as demonstrated for a non-maximally entangled state $(|\alpha|<|\beta|)$, our scheme can circumvent even ESD-causing conditions: for the weak measurement strength $p$ larger than a certain value, the two-qubit state $\rho_{\mathrm{r}}$ exhibits a positive concurrence $C_{\mathrm{r}}$, which means that Bob and Charlie can now share entanglement even through quantum channels of severe decoherence.

As the weak measurement and the reversal measurement are non-unitary operations, our scheme naturally has less than unity success probability ${ }^{25,26}$. As shown in Fig. $4 \mathrm{~b}$, the higher the concurrence $C_{\mathrm{r}}$, the larger the weak measurement strength $p$. We can then explore the trade-off relation between the success probability and weak measurement strength $p$. For a single qubit, the overall success probability can be calculated by averaging the state-dependent success probability for all states on the Bloch sphere and is given by $\mathcal{P}_{1}=(1 / 2) \bar{D} \bar{p}(2+D \bar{p})($ refs 25,26$)$. For the two-qubit state, the success probability is calculated to be

$$
\mathcal{P}_{2}=\left(\mathcal{P}_{1}\right)^{2}=\frac{1}{4} \bar{D}^{2} \bar{p}^{2}(2+D \bar{p})^{2}
$$

We have measured $\mathcal{P}_{2}$ by averaging the success probabilities for four two-qubit states, $|00\rangle_{\mathrm{S}},|01\rangle_{\mathrm{S}},|10\rangle_{\mathrm{S}}$ and $|11\rangle_{\mathrm{S}}$. Owing to the symmetry, averaging over the four two-qubit states is sufficient to obtain $\mathcal{P}_{2}$ experimentally. Figure 5 shows the experimentally obtained success probability as a function of weak measurement strength $p$. Clearly, the larger the weak measurement strength $p$, the less the success probability. In the asymptotic limit of $p \rightarrow 1$, concurrence $C_{\mathrm{r}}$ can be arbitrarily close to the initial value but $\mathcal{P}_{2} \rightarrow 0$.

We have demonstrated that weak measurement and quantum measurement reversal can indeed be useful for battling against 
decoherence. In particular, for amplitude-damping decoherence, we have shown that our protocol can distribute (protect) entanglement even through (from) severe decoherence. We have also studied the trade-off relation between the success probability, concurrence and weak measurement strength. Although the demonstration in this work was done for two-photon polarization qubits, the protocol can easily be applied to other types of qubit, making weak measurement and quantum measurement reversal powerful tools for battling against decoherence. We thus believe that it should be possible to effectively handle decoherence in quantum information by combining the scheme for protecting entanglement from decoherence discussed in this paper and entanglement distillation.

\section{Methods}

State preparation. First, the two-qubit maximally entangled state $\left(|\Phi\rangle=\alpha|00\rangle_{\mathrm{S}}+\beta|11\rangle_{\mathrm{S}}\right.$ with $\left.|\alpha|=|\beta|\right)$ is generated using type-I frequency-degenerate spontaneous parametric down-conversion from a 6 -mm-thick $\beta-\mathrm{BaB}_{2} \mathrm{O}_{4}$ crystal pumped by a $405 \mathrm{~nm}$ diode laser $^{28}$. The photons are frequency filtered with a set of interference filters with a $5 \mathrm{~nm}$ passband. The non-maximally entangled states $\left(|\Phi\rangle=\alpha|00\rangle_{\mathrm{S}}+\beta|11\rangle_{\mathrm{S}}\right.$ with $\left.|\alpha| \neq|\beta|\right)$ are generated by preferential amplitude reduction of one of the basis states using a set of glass plates oriented at the Brewster angle $\mathrm{e}^{13,24,26}$ (BPs).

Weak measurement and reversing measurement. The weak measurement and the reversing measurement for the single-photon polarization qubit are implemented with BPs and wave plates ${ }^{24}$. As the BP probabilistically rejects vertical polarization $\left(|1\rangle_{S}\right)$ and completely transmits horizontal polarization $\left(|0\rangle_{S}\right)$, a single-photon polarization qubit found behind a BP had been subject to weak measurement or partial collapse measurement towards the $|0\rangle_{\mathrm{S}}$. The reversing measurement is designed to reverse the effect of weak measurement by making partial collapse measurement towards the $|1\rangle_{s}$ and it can be implemented by adding $45^{\circ}$ half-wave plates (HWPs) before and after the BPs. The weak measurement and the reversing measurement strength $p$ and $p_{\mathrm{r}}$ can be varied by changing the number of BPs.

Amplitude-damping decoherence. The decoherence map of equation (1) causes state-dependent coupling of the system qubit (the single-photon polarization state) to the environment qubit (the single-photon path qubit) and is realized with an interferometer shown in Fig. 3 (ref. 26). The displaced Sagnac interferometer implements the coupling of the polarization qubit to the path qubit. The horizontal polarization $|0\rangle_{S}$ entering the polarizing beam splitter (PBS) can be found only at the $|0\rangle_{\mathrm{E}}$ output mode. The vertical polarization $|1\rangle_{\mathrm{S}}$ at the input of the PBS can be found both at $|0\rangle_{\mathrm{E}}$ and $|1\rangle_{\mathrm{E}}$ output modes according to the angle $\theta$ of the HWP. The probability that the vertical polarization $|1\rangle_{S}$ at the input of the PBS ends up at the $|1\rangle_{\mathrm{E}}$ output mode of the PBS corresponds to decoherence $D$ in equation (1) such that $\sqrt{D}=\sin 2 \theta$. As we are interested only in the system qubit, we need to trace out the environment qubit once the coupling is done. We realize the tracing out of the environment qubit by incoherently mixing $|1\rangle_{\mathrm{E}}$ (horizontally polarized) and $|0\rangle_{\mathrm{E}}$ (vertically polarized) at another beam splitter (BS) with a path-length difference sufficiently larger than the coherence length $(\sim 140 \mu \mathrm{m})$ of the single photon.

\section{Received 23 September 2011; accepted 21 November 2011; published online 18 December 2011}

\section{References}

1. Zurek, W. H. Decoherence, einselection, and the quantum origins of the classical. Rev. Mod. Phys. 75, 715-775 (2003).

2. Yu, T. \& Eberly, J. H. Finite-time disentanglement via spontaneous emission. Phys. Rev. Lett. 93, 140404 (2004).

3. Almeida, M. P. et al. Environment-induced sudden death of entanglement. Science 316, 579-582 (2007).

4. Nielsen, M. \& Chuang, I. Quantum Computation and Quantum Information (Cambridge Univ. Press, 2000).

5. Masanes, L., Pironio, S. \& Acin, A. Secure device-independent quantum key distribution with causally independent measurement devices. Nature Commun 2, 238 (2011).
6. Bennett, C. H. et al. Teleporting an unknown quantum state via dual classical and Einstein-Podolsky-Rosen channels. Phys. Rev. Lett. 70, 1895-1899 (1993).

7. Bouwmeester, D. et al. Experimental quantum teleportation. Nature 390, 575-579 (1997).

8. Kim, Y-H., Kulik, S. P. \& Shih, Y. H. Quantum teleportation of a polarization state with a complete Bell state measurement. Phys. Rev. Lett. 86, 1370-1373 (2001).

9. Giovannetti, V., Lloyd, S. \& Maccone, L. Advances in quantum metrology Nature Photon. 5, 222-229 (2011).

10. Bennett, C. H., Bernstein, H. J., Popescu, S. \& Schumacher, B. Concentrating partial entanglement by local operations. Phys. Rev. A 53, 2046-2052 (1996).

11. Bennett, C. H. et al. Purification of noisy entanglement and faithful teleportation via noisy channels. Phys. Rev. Lett. 76, 722-725 (1996).

12. Pan, J-W., Gasparoni, S., Ursin, R., Weihs, G. \& Zeilinger, A. Experimental entanglement purification of arbitrary unknown states. Nature 423, 417-422 (2003)

13. Kwiat, P. G., Barraza-Lopez, S., Stefanov, A. \& Gisin, N. Experimental entanglement distillation and 'hidden' non-locality. Nature 409, 1014-1017 (2001).

14. Dong, R. et al. Experimental entanglement distillation of mesoscopic quantum states. Nature Phys. 4, 919-923 (2008).

15. Gühne, O. \& Tóth, G. Entanglement detection. Phys. Rep. 474, 1-75 (2009).

16. Lidar, D. A., Chuang, I. \& Whaley, K. B. Decoherence free subspaces for quantum computation. Phys. Rev. Lett. 81, 2594-2597 (1998).

17. Kwiat, P. G., Berglund, A. J., Alterpeter, J. B. \& White, A. G. Experimental verification of decoherence-free subspaces. Science 290, 498-501 (2000).

18. Facchi, P., Lidar, D. A. \& Pascazio, S. Unification of dynamical decoupling and the quantum Zeno effect. Phys. Rev. A 69, 032314 (2004).

19. Maniscalco, S., Francica, F., Zaffino, R. L., Lo Gullo, N. \& Plastina, F. Protecting entanglement via the quantum Zeno effect. Phys. Rev. Lett. 100, 090503 (2008).

20. Williams, N. S. \& Jordan, A. N. Entanglement genesis under continuous parity measurement. Phys. Rev. A 78, 062322 (2008).

21. Koashi, M. \& Ueda, M. Reversing measurement and probabilistic quantum error correction. Phys. Rev. Lett. 82, 2598-2601 (1999).

22. Korotkov, A. N. \& Jordan, A. N. Undoing a weak quantum measurement of a solid-state qubit. Phys. Rev. Lett. 97, 166805 (2006).

23. Katz, N. et al. Reversal of the weak measurement of a quantum state in a superconducting phase qubit. Phys. Rev. Lett. 101, 200401 (2008).

24. Kim, Y-S., Cho, Y-W., Ra, Y-S. \& Kim, Y-H. Reversing the weak quantum measurement for a photonic qubit. Opt. Express 17, 11978-11985 (2009).

25. Korotkov, A. N. \& Keane, K. Decoherence suppression by quantum measurement reversal. Phys. Rev. A 81, 040103(R) (2010).

26. Lee, J-C., Jeong, Y-C., Kim, Y-S. \& Kim, Y-H. Experimental demonstration of decoherence suppression via quantum measurement reversal. Opt. Express 19, 16309-16316 (2011).

27. Wootters, W. K. Entanglement of formation of an arbitrary state of two-qubits. Phys. Rev. Lett. 80, 2245-2248 (1998).

28. Shih, Y. H. \& Alley, C. O. New type of Einstein-Podolsky-Rosen-Bohm experiment using pairs of light quanta produced by optical parametric down conversion. Phys. Rev. Lett. 61, 2921-2924 (1988).

\section{Acknowledgements}

This work was supported by the National Research Foundation of Korea (2009-0070668 and 2011-0021452).

\section{Author contributions}

Y-S.K. and J-C.L. carried out the theoretical calculations, designed and carried out the experiment, analysed data and drafted the manuscript. O.K. carried out the experiment. Y-H.K. conceived the idea, designed the experiment, analysed data, wrote the manuscript and supervised the project.

\section{Additional information}

The authors declare no competing financial interests. Reprints and permissions information is available online at http://www.nature.com/reprints. Correspondence and requests for materials should be addressed to Y-H.K. 